\title{
LAS CIENCIAS SOCIALES COMO CONOCIMIENTO DE LA ÉPOCA
}

Rodrigo Baño Ahumada 


\section{RODRIGO BAÑO AHUMADA}

Abogado, Licenciado en Sociología, profesor asociado de la Universidad de Chile. Áreas de interés: Sociología política, sociología del Estado, análisis político y movimientos sociales. 


\section{LAS CIENCIAS SOCIALES COMO CONOCIMIENTO DE LA ÉPOCA}

\section{INTRODUCCIÓN}

Todo pensamiento es pensamiento de su época. Más allá de las connotaciones que tiene la frase en las complejidades de la filosofía hegeliana, la clásica expresión pareciera dar cuenta de lo natural y obvio, pero, como suele suceder con lo obvio, no se le considera en todo su alcance. Por el contrario, regularmente se hace el estudio de las ideas como si las ideas tuvieran la capacidad de engendrar ideas, sin que tenga mayor importancia lo que esté ocurriendo en la época. Los científicos sociales y particularmente los sociólogos suelen relacionar los problemas que estudian con el contexto en que estos se presentan, pero muy pocas veces tienen conciencia de que las teorías y métodos que usan en sus estudios son pensamientos de su época. Alguna vez se desarrolló una sociología del conocimiento, que pretendía entender este en relación con el contexto social en que se producía, pero nunca existió una sociología del conocimiento de la sociología. No es tan raro: solemos creer en la verdad de lo que planteamos como conocimiento. La tierra fue indudablemente plana durante muchos siglos; hoy afirmamos con el mismo énfasis que es redonda.

Cuando se plantea el tema de evaluar lo que significó para las ciencias sociales, especialmente para su desarrollo en la Universidad de Chile, el Golpe de Estado de 1973 y el régimen dictatorial que se instauró por largos 17 años, es natural que se piense inmediatamente en lo que ese régimen significó en cuanto persecución y represión extendida y brutal sobre quienes desarrollaban tales ciencias sociales. También se tiene muy presente el impacto directo que este régimen tuvo sobre el ejercicio de tales ciencias sociales, puesto que se limitó su enseñanza, se redujo su presupuesto y se dificultó enormemente las posibilidades de investigar y publicar sobre sus propios temas. Efectivamente, eso es innegable y existe abundante información al respecto, aunque ella no cuente con la difusión que debería haber tenido y no se le considera con la fuerza que debiera considerarse. Es ineludible hacer referencia a esto, pero también parece necesario abordar la transformación que experimentó la ciencia social y especialmente la sociología.

Al efecto, creo conveniente cuestionar la apreciación de que el Golpe militar significó un duro golpe al desarrollo de las ciencias sociales. No para negar que hubiera un ataque a las ciencias sociales y a quienes la practicaban, sino para negar la idea del desarrollo de las ciencias como un proceso evolutivo que se va implementando en las sociedades, a imagen y semejanza del desarrollo de la razón en cierta filosofía. A sabiendas de lo difícil que es eludir el evolucionismo instalado confortablemente en el sentido común, prefiero plantear más una diferenciación cualitativa que cuantitativa. No es que las ciencias tengan un mayor o menor desarrollo a través del tiempo, desde una perspectiva evolucionista que siempre plantea el presente como una etapa superior del pasado y vislumbran el futuro como un horizonte de mayor superación, sino que las ciencias 
cambian. Carece de sentido pensar que un momento histórico determinado tenga superioridad sobre otro momento histórico, a menos que imaginemos un absoluto con lo cual se pueda establecer la comparación en términos de mayor o menor proximidad, como hace la filosofía de la historia, que plantea un punto de llegada de esta a partir del cual se puede evaluar la distancia a que se está en cada momento.

Lejos de tal pretensión evolucionista, lo que interesa aquí será precisamente comparar distintos momentos históricos de las ciencias sociales en términos de las características que permiten establecer diferenciaciones.

\section{SITUACIÓN DE LAS CIENCIAS SOCIALES AL QUIEBRE INSTITUCIONAL}

Obviamente, uno de los primeros problemas con que se enfrenta la pretensión de hablar de las ciencias sociales es delimitar qué se entiende por ciencias sociales y, en consecuencia, cuáles son sus disciplinas. Como suele ocurrir, toda definición es pretenciosa y arbitraria, por más que se intente justificarla racionalmente, de manera que es mejor prescindir de ello y, consecuentemente, abandonar también todo intento de establecer cuáles disciplinas abarca. Simplemente utilizaré como referencia directa la sociología y tangencialmente otras disciplinas que actualmente se agrupan en la Facultad de Ciencias Sociales, lo cual, obviamente, no significa negar el carácter social de disciplinas como la economía, la historia y otras. Valga como excusa mi ignorancia sobre disciplinas que exceden mis ya limitados conocimientos sobre sociología.

La existencia de las disciplinas agrupadas actualmente en la Facultad de Ciencias Sociales es bastante breve, pues ellas nacen institucionalmente solo a mediados del siglo XX. En aquellos tiempos, en que bastaba decir "la Universidad" para que todos entendieran que se estaba hablando de la Universidad de Chile, la psicología y la sociología comenzaron desarrollándose como institutos de investigación para rápidamente derivar hacia la creación de escuelas de pregrado, siendo pioneras no solo en Chile sino que de las primeras en América Latina. También se crea el Centro de Estudios Antropológicos, que en 1962 empieza a dictar cursos de especialización en antropología a estudiantes de Historia, y que a comienzos de la década de los 70 dará lugar a una licenciatura en antropología.

A partir de esos comienzos, esas disciplinas de ciencias sociales tendrán un vigoroso crecimiento en nuestra Universidad, acompañadas luego por su establecimiento en la Universidad Católica y Universidad de Concepción. Aumenta el número de estudiantes, aumenta el número de profesores y aumentan las investigaciones y publicaciones.

Este proceso es a menudo descrito posteriormente en términos de etapas en que las disciplinas adoptan distintas orientaciones y énfasis. En el caso específico de la sociología, se habla también de etapas: al comienzo habría lo que se llama "sociología de cátedra", practicada desde antes que existiera una carrera institucionalizada, a través de la exposición y desarrollo de los grandes pensadores. Luego vendría una etapa de sociología, denominada a menudo "profesional", caracterizada por su orientación funcionalista y empirista, que se nutre de sociólogos formados en Estados Unidos o bajo 
su influencia. El tercer período es caracterizado como etapa "ideológica", la que correspondería a una crítica al modelo anterior y al intento de desarrollar la sociología de acuerdo al modelo marxista. A nadie escapará, puesto que todos son muy sagaces, que la denominación de "ideológica" que se da a la tercera etapa por algunos autores deriva de la ideología de tales autores, puesto que las orientaciones de la sociología son siempre ideológicas o no lo son nunca; elija usted. La pretensión, muy de moda, de que en economía, en política y en sociología se ha "superado" un pasado ideológico para llegar a un presente técnico, pragmático y objetivo, puede ser muy valiosa para desarrollar la autoestima personal y la de una época, pero es bastante ridícula.

Sea como sea, que no puede ser de otra manera, al momento del Golpe militar de 1973, encontramos una sociología y unas ciencias sociales en pleno auge, valorizadas como nunca antes y con presencia en prácticamente toda la Universidad. Es, naturalmente, la Universidad de Chile la que va a la vanguardia de este desarrollo de las ciencias sociales. Sus investigaciones tienen inicialmente su sede en el Instituto de Sociología (creado en 1946) y en el Instituto Central de Psicología (creado en 1941), y posteriormente derivan hacia la formación de psicólogos en 1948 y de sociólogos en 1957. También es en la Universidad de Chile donde se inicia la formación de antropólogos en 1971. Es precisamente en la Universidad de Chile donde se desarrolla no solo la investigación y docencia en ciencias sociales al momento del Golpe militar, sino que es el lugar donde se plantea el debate académico y político sobre estas disciplinas.

El Golpe militar y el régimen que este instauró, no solo significó en nuestra Universidad la represión brutal sobre muchos de los que cultivaban las ciencias sociales, sino que además la eliminación de su capacidad de investigación, la limitación y control de su docencia, y su confinamiento a una situación de deterioro y aislamiento. Sin embargo, como ya se ha señalado, no es ese el tema que interesa desarrollar aquí, sino las consecuencias que la intervención militar tuvo en cuanto redefinición de la situación y orientación de las ciencias sociales, y del lugar que en esto ocupó la Universidad de Chile.

\section{REDEFINICIÓN DE LA SITUACIÓN POR LA ACCIÓN REPRESIVA}

Es bien sabido, pero hay que repetirlo igual: el Golpe militar marca el inicio de cambios radicales en las tendencias que vivía hasta el momento el país en todos sus ámbitos. Naturalmente, la educación, las universidades y en especial las ciencias sociales sufrirían también transformaciones substanciales.

La violencia con que se hicieron esos cambios es bien conocida y hoy está documentada al menos en sus aspectos más notorios, como son los fusilamientos, las torturas, las prisiones, las relegaciones y expulsiones, y las más de mil cien personas detenidas y hechas desaparecer entre el 11 de septiembre de 1973 y el 11 de marzo de 1990. Las víctimas que declararon ser profesionales/técnicos y estudiantes, representan algo más de un $15 \%$ para cada uno de ellos ( 4.174 y 4.114 respectivamente) $)^{1}$. Entre estos últimos, se

1. Comisión Nacional de Verdad y Reconciliación, Colección Archivo Nacional de Chile, Santiago, 1991. 
sabe que los científicos sociales fueron particularmente afectados. Hubo persecución de sociólogos y estudiantes de sociología destacados, y se sabe que varios fueron detenidos desaparecidos, fusilados y torturados, desde el mismo 11 de septiembre de 1973.

Las universidades sufren el Golpe de Estado y posteriormente las consecuencias de la represión, y la Universidad de Chile será la más afectada. El Golpe militar significará para nuestra Universidad, más allá de la represión inmediata, un ataque continuo que pareció siempre orientado a su total desaparición. Sobrevive dramáticamente reducida, al ser despojada de todas sus sedes regionales y de su gran Pedagógico, junto con verse obligada a privatizarse en los hechos debido a la pérdida de financiamiento fiscal y a la necesidad de autofinanciarse. En el comienzo, la sede Oriente de la Universidad de Chile, donde están fundamentalmente las ciencias sociales, es allanada y ocupada por las fuerzas militares, sus oficinas saqueadas y la biblioteca de Periodismo incendiada por las tropas. Profesores, administrativos y estudiantes son detenidos, torturados, muertos. Una veintena de profesores universitarios son fusilados los primeros 45 días $^{2}$. Así como el bombardeo de la Moneda fue el anuncio del despiadado régimen que se instalaba, la fuerte represión inicial en la Universidad de Chile fue el anuncio del serio intento de eliminarla, que finalmente terminaría en un grave deterioro aún no reparado.

Pero no solo hubo esta represión directa, sino que muchos de los que la sufrieron fueron expulsados del país, o tuvieron que exiliarse ante una amenaza o simplemente porque se vieron privados de sus fuentes de trabajo. Esta situación afectó gravemente a quienes se dedicaban a estudiar o cultivar las ciencias sociales y, particularmente, a los sociólogos. Aquellos que pudieron permanecer en el país tuvieron que orientar sus actividades hacia aquellas que el régimen militar permitía, entre las cuales no estaba el desarrollo de la libertad de pensamiento ni los estudios con espíritu crítico, al cual los gobernantes eran especialmente sensibles.

Como corresponde a una represión masiva y bien organizada, muy pronto se dictaron medidas dirigidas directamente contra las instituciones y, tras el Golpe militar, la iniciativa de las nuevas autoridades fue intervenir radicalmente todas las universidades chilenas. Esto tenía como finalidad obtener el control político de lo que es considerado el espacio en que las sociedades se piensan a sí mismas.

La educación siempre ha sido tema de preocupación en todas las sociedades que se conocen, pues es la forma en que estas se mantienen y reproducen. Se transmiten los conocimientos y las pautas de orientación valorativas, aunque esto se realice inicialmente de manera intuitiva. Las sociedades no viven sin historia, así como los sujetos individuales no viven sin memoria. Considerando, además, que el pasado no es antecedente del presente, sino que es su componente: el adulto no deja de ser el niño que fue, así como las sociedades no dejan de ser el pasado que tuvieron. Pero la educación no solo apunta a la reproducción de las sociedades y de los individuos, sino que también establece los fundamentos de la proyección de sociedades e individuos. Historia y utopía.

2. Arzobispado de Santiago, Fundación de documentación y archivo de la Vicaría de la Solidaridad. Capítulo quinto: "La situación laboral". No hay más referencias. 
En la medida que las sociedades pierden su ingenuidad, toman conciencia y racionalizan sus acciones, la educación pone más el acento en el proyecto que en la historia. El pensamiento clásico griego y especialmente Platón, desarrollará la convicción de que el ser humano tiene plasticidad para ser moldeado, que puede formarse al ciudadano en una orientación determinada. Gran parte de La República de Platón está destinada a explicitar el hecho de que la educación es el instrumento para moldear el buen ciudadano.

Las instituciones educacionales de la época moderna tienen el mismo supuesto, esto es, que es posible formar al ser humano de determinada manera. Dichas instituciones se desarrollarán entonces con esa pretensión de educar, pero es necesario tener en cuenta que también históricamente cambiarán los contenidos y la manera en que se pretende formar.

Se comprende, entonces, la preocupación del nuevo Gobierno, impuesto a través de un Golpe militar, de controlar el pensamiento y por tanto de obtener el control político de las instituciones encargadas de formar a los ciudadanos, lo que resulta particularmente importante a nivel de las universidades, que no solo comunican conocimiento, sino que también lo crean. Además, que en la época, las universidades habían desarrollado un proceso de democratización interna que otorgaba mayor participación a los miembros de la comunidad universitaria.

Se dicta muy pronto, el 2 de octubre de 1973, el Decreto Ley $N^{\circ} 50$ que suprime la autonomía universitaria y toda forma de participación interna y que se orienta a la depuración drástica de los claustros universitarios de académicos y estudiantes. Se intervienen los planteles de educación superior con la designación de Rectores Delegados para las 8 universidades, los cuales serían los encargados de hacer limpieza en todo el estamento universitario. Estos fueron oficiales en servicio activo o en retiro de las Fuerzas Armadas, que tenían atribuciones y recursos para intervenir, modificar, crear, refundir o suprimir unidades académicas, departamentos, carreras; junto con poder remover o destituir personal académico, administrativo y estudiantes ${ }^{3}$.

Pocos días después de la entrada en vigencia del Decreto Ley № 50, el Rector Delegado de la Universidad de Chile designa 36 fiscales para cubrir los casos de sanciones al personal de las sedes de la Universidad. Estimaciones conservadoras indican que fue marginado alrededor del 30\% al 35\% del personal docente, $10 \%$ a $15 \%$ de personal no académico y entre $15 \%$ y $18 \%$ de los alumnos. Las ciencias sociales son las más afectadas por la reestructuración. De las expulsiones de la Universidad de Chile, 255 eran de la Facultad de Ciencias Sociales, 120 del Departamento de Educación, 160 de la Facultad de Filosofía y 212 del Departamento de Economía Política.

Proporcionalmente, esto se expresa con mayor claridad al señalar que, del total de exonerados, los porcentajes que afectan a las ciencias sociales son extraordinariamente altos (77\% del Departamento de Geografía y Cartografía, 55\% de la Facultad de Ciencias Sociales sede Oriente, 36\% de la Facultad de Filosofía, 23\% de la Facultad de Ciencias

3. Archivo Chile, Sumariados, "La dictadura y la educación", Disponible en www.archivochile.cl 
Jurídicas y Sociales). Esto considerando que se suprimen 450 jornadas completas en la Universidad de Chile y 120 en la Universidad Católica4.

Unidades completas fueron cerradas en la Universidad de Chile: el Centro de Estudios Socioeconómicos, la carrera de Servicio Social, el Centro de Estudios Estadísticos y Matemáticos, el Departamento de Lenguas Eslavas, la Unidad de Historia Económica y Social del Departamento de Historia, y una parte del Programa de Enfermeras del Departamento de Especialidades Médicas de la Escuela de Medicina, la Facultad de Economía Política de la sede Norte, los departamentos de Administración y Ciencias Sociales sede Temuco, y el Centro de Estudios Históricos y Filosóficos sede Valparaíso. Las vacantes en la Universidad de Chile en cinco años se vieron reducidas a la mitad (de 21.000 en 1973 a 11.000 en 1978), mientras la Universidad Católica prácticamente mantenía todas sus vacantes (3.500 en 1973, 3.400 en 1978).

En lo referido a las ciencias sociales, habría que señalar que no alcanzó a concretarse el decreto Universitario 4.513 que creaba la Facultad de Ciencias Sociales (que las trasladaría a Juan Gómez Millas), pues el Golpe de Estado frenó los procesos, quebrándose la administración naciente de la nueva facultad y estancándose el otro proyecto ya mencionado. En vez de aquello las carreras de Antropología, Arqueología y Sociología se fusionan en un solo departamento.

Grafica bastante la situación si se recuerda que en la carrera de Sociología, en 1973, se eliminó a 37 de los 40 profesores que había en su planta académica, resultando solo tres de ellos aceptables para las nuevas autoridades. La carrera, trasladada físicamente lejos de los centros universitarios y de la ciudad misma, logró subsistir con nuevas contrataciones de personal de confianza, llegando a tener en 1980 una planta de 12 profesores de tiempo completo 5 . No obstante, en 1981 cerró el ingreso de la carrera de nuevos alumnos, a pesar de que en 1980 postularon a la carrera alrededor de 900 candidatos en primera opción para 20 vacantes.

Las ciencias sociales no desaparecieron en Chile, pero claramente cambiaron. Al ser reprimidas, desfinanciadas y rigurosamente controladas en la Universidad de Chile, su investigación se detuvo y su docencia fue limitada y suspendida en algunos años, teniendo que reorientarse en función de la ideología impuesta por el régimen militar. Los trabajos en ciencias sociales y, particularmente, en sociología se trasladaron a otros terrenos y tomaron desarrollos y orientaciones diversos a los que prevalecían con anterioridad al Golpe militar.

\section{LA DIFÍCIL INSTITUCIONALIDAD DE LAS CIENCIAS SOCIALES}

El proceso represivo en las ciencias sociales resultó devastador en las universidades en general, pero fue particularmente fuerte en la Universidad de Chile. Aunque subsistió la

\footnotetext{
4. Garretón, M.A., Las Ciencias Sociales en Chile. Institucionalización, ruptura y renacimiento, véase www.manuelantoniogarreton.cl/ultimoslhtml

5. Ibíd
} 
carrera de sociología, esta fue drásticamente cambiada en sus contenidos y orientaciones, eliminándose totalmente su capacidad de investigación.

En la Universidad Católica la represión a las ciencias sociales fue también dura, aunque más matizada. Se cierran algunas unidades, como el Centro de Estudios de la Realidad Nacional, el Programa de Estudios y Capacitación Laboral y la carrera de Sociología en la Universidad Católica fue inicialmente suspendida. No obstante, su Instituto de Sociología fue la institución universitaria cuya producción académica adquirió mayor influencia científica y social en ese ámbito, realizando el paso de un canon centrado en el concepto de estructura social hacia otro que enfatiza el concepto de cultura, asumida esta como un sistema de valores. También será en la Universidad Católica donde en plena dictadura, en el año 1979, se funda el Instituto de Ciencia Política, que realiza una amplia labor inspirada en la ciencia política norteamericana. María de los Ángeles Fernández, presenta el siguiente punto de vista respecto al período del régimen militar:

Se reconoce que, en ciertas condiciones, la ciencia política puede desarrollarse sin traumas bajo un régimen autoritario, como fue el caso de Chile. Efectivamente, las instituciones más antiguas de la disciplina se asientan en los años del régimen militar e inician sus programas de Magíster. Su desarrollo en democracia también puede verse en aprietos: no en vano se recuerda cómo fue abortada por los efectos de la polarización ideológicas de la década de los años 60. Durante los años 80, hubo mucha actividad, expresada en publicaciones, dada la disponibilidad de recursos de organismos extranjeros y la clausura de la docencia, que permitía tiempo para la investigación, pero se duda de si eso representa efectivamente autonomía. Se habla de ese tiempo como de uno de "autonomía relativa" para la ciencia política ${ }^{6}$.

Suprimido prácticamente el desarrollo de las ciencias sociales en las universidades, los estudios sobre la materia se trasladarán a otros espacios. Entre ellos, cobran importancia las llamadas organizaciones no gubernamentales (ONG), que se instalarán fundamentalmente a partir de la segunda mitad de la década de los 70 . También existen organismos internacionales que adquirirán una nueva función, como ocurre particularmente con la Facultad Latinoamericana de Ciencias Sociales (FLACSO). Ahí se desarrollará la cultura opositora, nutriéndose fundamentalmente de profesionales expulsados de las universidades y que pudieron permanecer o regresar a Chile.

Antes de 1973 existían varios centros independientes vinculados a la Iglesia Católica y a la Democracia Cristiana, como son el CIAS, Instituto de Humanismo, Centro para el Desarrollo Económico y Social de América Latina (DESAL), Centro Latinoamericano de Población y Familia (CELAP), Centro de Investigación y Desarrollo de la Educación (CIDE), Instituto Latinoamericano de Doctrina y Estudios Sociales (ILADES), Instituto de Estudios Políticos (IDEP). Además, funcionaban organismos internacionales como CEPAL, FLACSO e ICIRA.

6. Fernández, M. de los A., "Ciencia Política en Chile: Un espejo intelectual”, en Revista de Ciencia Política, 25 (1), Pontificia Universidad Católica de Chile, 2005. 
Durante la época de dictadura y en reacción a la situación que se vivía en las universidades, surgen muchas ONG o centros académicos independientes (CAI), fuera del sistema universitario, que alojan el trabajo de intelectuales de las ciencias sociales. Estos, formaron parte de la cultura opositora, aunque también algunos tienen coloración oficialista y trabajan principalmente en función de temas, áreas, problemas u objetos especializados. Para mostrar su número y diversidad se puede nombrar a los siguientes por orden de aparición: 1974, Instituto Chileno de Estudios Humanísticos (ICHEH), ligado a la DC; 1975, Academia de Humanismo Cristiano, del Arzobispado de Santiago (que en la década de los 90 se transformará en Universidad); 1978, Programa de Economía del Trabajo (PET); 1978, Grupo de Investigaciones Agrarias (GIA); 1978, Grupo de Estudios Agro regionales (GEA); 1979, Programa de Estudios sobre la condición de la mujer; 1976, Corporación de Investigaciones Económicas para América Latina (CIEPLAN), antes vinculado al Centro de Estudios de Planificación (CEPLAN) de la Universidad Católica; 1977, Programa de Investigaciones Interdisciplinarias en Educación (PIIE), que anteriormente era de la PUC, se incorpora a la Academia de Humanismo Cristiano (AHC); 1978, Corporación de Investigaciones para el desarrollo (CINDE); 1979, SUR; 1979, Centro de Investigación y Planificación del Medio Ambiente (CIPMA); 1979, Grupo Educación y Comunicaciones (ECO); 1979, Programa Interdisciplinario de Investigación, Enseñanza y Difusión de Derechos Humanos; 1980 ,Centro de Estudios Públicos (CEP), área liberal y financiamiento empresarial; 1980, se instala la sede del Instituto Latinoamericano de Estudios Transnacionales (ILET); 1980, Centro para el Desarrollo Campesino y Alimentario (AGRARIA); 1981, Centro de Estudios del Desarrollo (CED)

El conjunto de estas instituciones forma a partir de 1980 lo que se ha denominado en Chile un sector académico independiente o informal, que hacia fines de 1985 se hallaba integrado por alrededor de 40 centros privados de investigación en ciencias sociales. Trabajaban en ellos hacia esa misma fecha un total de 543 investigadores, excluidos los ayudantes y becarios, de los cuales alrededor de un 30\% poseía estudios de postgrado a nivel de doctorado o maestría ${ }^{7}$.

Otro aspecto importante, y que tendrá también una fuerte influencia en el desarrollo de las ciencias sociales en los años 70 y 80 , será el financiamiento de estos centros independientes. Negado este por los respectivos gobiernos nacionales, debió buscarse en fuentes internacionales, principalmente en América del Norte y en Europa. Entre las agencias que financiaron estas actividades, se encuentran las tradicionales Fundación Ford y Rockefeller más la fundación Tinker de USA, el International Development Research Center (IDRC) de Canadá, La Swedish Agency for Research Cooperation (SAREC) de Suecia y

7. Brunner, J.J., Barrios, A., El caso de la sociología en Chile. Formación de una disciplina, Santiago: FLACSO, 1988, págs. 242-243. 
un conjunto de otras agencias como NOVIB y CEBEMO de Holanda, Paz y Desarrollo de Canadá, el CCFD de Francia y la Fundación Interamericana de USA

De todas estas instituciones, la que alcanzó mayor relieve fue FLACSO, que siendo inicialmente un organismo internacional con sede en Chile, pudo sobrevivir al Golpe militar, aunque no pudo continuar con sus maestrías en sociología y ciencia política, las que tuvieron que trasladarse inicialmente a una sede creada en México y posteriormente a sedes en varios países latinoamericanos. Posteriormente, en 1979, el Gobierno de Pinochet desahució el tratado internacional y FLACSO en Chile se cobijó bajo el alero de la Academia de Humanismo Cristiano, que tenía la protección del Arzobispado de Santiago. Como señala un autor:

Aunque en más de una ocasión FLACSO-Chile hubo de enfrentar dificultades con el gobierno militar, pudo convertirse en un "refugio" para quienes quedaron en ese país, y no sólo continuó sino que incrementó progresivamente su trabajo investigativo llegando a convertirse hacia los 80 en uno de los centros más importantes de la región ${ }^{9}$.

Efectivamente, dedicada fundamentalmente a investigación y publicaciones, FLACSO fue el organismo que más influyó en el giro que tomaron las ciencias sociales y con el retorno a la democracia varios de sus miembros ocuparon los cargos más relevantes del poder político.

Es importante señalar que los científicos sociales de la Universidad de Chile, más diezmados y perseguidos, fueron en general minoritarios tanto en la FLACSO como en otras ONG, donde la hegemonía la tuvieron los que provenían de la Universidad Católica. Situación que contribuye a explicar, junto con otros factores, la mayor dificultad que experimentaría la Universidad de Chile cuando, con el retorno a la democracia, empieza a tratar de revitalizar su desarrollo en ciencias sociales.

\section{LA REORIENTACIÓN DE LAS CIENCIAS SOCIALES: TEMAS Y ESTILOS}

Sabemos que en ciencias sociales es pretensioso plantear relaciones causales y que, a lo más, solo podemos intentar establecer relaciones entre variables, sabiendo que un determinado fenómeno admite muchas relaciones entre variables que concurren a su explicación. En consecuencia, sería absurdo pretender atribuir al Golpe militar y al régimen que este impuso los cambios ocurridos en los temas de estudio y en los estilos de análisis. Más aun, una somera comparación internacional permitiría comprobar que los cambios de temas y estilos no solamente suceden en Chile, sino que en muchas

8. Vasconi, T.A., Las ciencias sociales en América del Sur y Chile, 1960-1990, Working Paper Centro de Investigaciones Sociales, Universidad ARCIS, 1996, pág. 45.

9. Ibíd., pág. 43. 
otras partes donde no ocurrieron similares acontecimientos y procesos políticos. Sin embargo, más allá de esta generalidad innegable, es necesario considerar que la forma, profundidad y extensión con que en Chile se producen estos cambios, alguna relación parecen tener con las características de su proceso político. Al respecto, parece conveniente señalar que las transformaciones ocurridas no han dejado de ser señaladas por diversos autores, aunque haya algunas divergencias acerca de las características de estas.

El profesor Garretón, en su programa para el curso de Teoría Sociológica V, señalará que en esos momentos de crisis, provocados por la intervención militar, los rasgos de la reflexión sociológica serán, además de la pérdida de bases institucionales por la intervención de las universidades, la ausencia de categorías y datos básicos, la identificación de denuncia y comprensión, la debilidad de la teoría básica y el abandono de los paradigmas globalizantes; señala también la evolución temática: nuevas formas de dominación, fascismo, militarismo; el carácter del Estado; la resurrección de la sociedad civil; el neoliberalismo, los nuevos actores y movimientos sociales; regímenes políticos, transiciones y democratizaciones, identidad cultural y modernidad.

Por su parte, el análisis de discurso realizado por Alvarado y Santander ${ }^{10}$ muestra "el paso desde el canon centrado en el concepto de estructura social hacia otro que enfatiza el concepto de cultura, asumida esta como un sistema de valores". También señala que en los primeros años después de 1973, tanto en el interior como en el exterior del país, los estudios continúan la línea anterior de corte marxista, aunque ahora abordando el fracaso del proyecto socialista que tenía la Unidad Popular en Chile. Posteriormente los estudios se enfocan fundamentalmente en el cuestionamiento crítico del modelo político dictatorial implantado, y la sociología se ve orientada hacia estudios de la realidad social chilena en función de la restauración de la democracia y los procesos que se encausan para obtenerla, reduciéndose fundamentalmente a publicaciones sobre el proceso de democratización. La reformulación de los estudios se corresponde con la emergencia del canon centrado en el concepto de cultura y bajo esa cobertura se abordan tópicos como los de etnicidad, identidad, género, jóvenes, territorialidad, sujeto, actor, etc. Según los autores se generaría una crisis de paradigma y surgirán nuevos modos de interpretación y nuevas estrategias metodológicas para reemplazar los esquemas de la sociología funcionalista y materialista histórica precedente.

Tomás Vasconi, en el análisis ya citado del período 1960-1990, también señala que los elementos fundamentales que enmarcaron la problemática e impusieron una óptica particular a las investigaciones desde los años 60, fueron progresivamente desapareciendo.

Uno de ellos será lo que hemos llamado el latinoamericanismo, esto es, el esfuerzo permanente por incluir los problemas que se analizaban, que se estudiaban y discutían, en una perspectiva regional. El otro -que no fue patrimonio exclusivo de los analistas sociales latinoamericanos sino también de europeos y norteamericanos a

10. Alvarado, M., Santander, P., "Matar al padre: Análisis discursivo de dos textos de la sociología chilena en período de dictadura", en Literatura y Lingüística, 14, UCSH, 2003. 
partir de la última posguerra- fue el "tercermundismo". Las investigaciones fueron volviéndose cada vez más "nacionales"; y aún más, especializándose temáticamente: el Estado, los partidos políticos, los movimientos sociales, la mujer, etc. ${ }^{11}$

Se podrían, al efecto, hacer muchas otras citas, no obstante prefiero, como el bueno de Walt, cantarme a mí mismo y presentar lo que respecto a este asunto escribí hace ya bastante tiempo. De hecho, ya en 1984, con una capacidad de predicción que ya me gustaría tener cuando apuesto en las carreras de caballos, señalo los cambios en los temas y estilos que tienden a establecerse en sociología. La ocasión fue una ponencia, presentada al Primer Congreso Chileno de Sociología, titulada "Nuevos Estilos y Nuevos Temas en el Análisis de Ciencias Sociales en la Última Década" y que posteriormente, en el mismo año, se publicó como Documento de Discusión de FLACSO ${ }^{12}$.

Al releer ahora este artículo tuve la tentación, tan nuestra, de hacer algunas modificaciones menores y publicarlo como un nuevo trabajo sobre la situación actual de las ciencias sociales. Sin embargo, como ya no necesito fabricar currículo, he preferido utilizarlo como material para referirme a aquella época. Como carezco de la modestia de los grandes hombres (siempre he sido bajo de estatura) me permito citar textualmente algunos párrafos:

Cuando se inicia la formación sistemática de sociólogos en Chile, a fines de la década del cincuenta, la sociología en boga reconoce una fuerte influencia norteamericana que le imprime un sello funcionalista y un marcado empirismo que se expresa en el reinado del survey para el análisis y búsqueda de solución para problemas concretos en campos especializados. Posteriormente viene toda una crítica al funcionalismo y a sus técnicas, que desembocan en la adopción de una línea "histórico estructural" que, a poco andar, lleva al predominio del análisis "marxista" que prácticamente redujo muchos estudios a una docta repetición de los conceptos fundamentales de Marx y Engels para deducir de ahí los conocimientos adecuados al tema propuesto.

Después del golpe militar de 1973 siguió predominando, por un corto tiempo ese estilo de "análisis marxista", pero luego derivó hacia consideraciones más globales y generales que, a partir de la crítica europea a los socialismos reales y al marxismo, condujo a intentos -muy ligados a la filosofía- de construir nuevas visiones de mundo y nuevos métodos de análisis referidos a grandes temas como el Estado, las clases sociales, relaciones entre economía y política, epistemología, lenguaje, etc.

Si contemplamos en la actualidad lo que se entiende como producción de los cientistas sociales, nos encontramos con un cuadro bastante diverso. Relegados los grandes temas a un segundo plano, ya casi nadie se preocupa de América Latina, el carácter del Estado, la definición de las clases sociales, la relación entre economía y política. Los sociólogos, confundidos con trabajadores sociales, educadores

11. Vasconi, T.A., Ibíd.

12. Baño, R., "Nuevos estilos y nuevos temas en el análisis de ciencias sociales en la última década", en Documento de Discusión, Nº 60, Programa FLACSO, Santiago, 1984. 
populares y periodistas, deambulan por las poblaciones, los sindicatos, caseríos rurales, mujeres femeninas y jóvenes juveniles, buscando captar y reproducir, lo más fielmente posible, la expresión propia de los actores. La grabadora de casette es la compañera fiel por esos campos.

Pero no sólo se trata de transformarse en papel de calco de la realidad. También se hacen cosas. Los más decididos construyen huertos familiares, inventan tecnologías apropiadas, forman grupos de mujeres, rehabilitan caídos.

Es evidente que algo ha pasado, que tales virajes no son meramente productos casuales de una imaginación sociológica febril. Más aún cuando hay una correspondencia tan estrecha entre los temas y estilos que adoptan los sociólogos y los temas y estilos que privilegian las agencias que los financian. Y, además, que el peso de las agencias financieras es total y absoluto en la medida que la sociología sufre, bajo el régimen autoritario, de la exclusión del financiamiento universitario y la profesión llega a ser casi delictual.

El análisis que se hace en ese texto sobre los cambios que ya habían ocurrido en los temas y estilos de análisis social se centra en tres consideraciones: la desaparición de la perspectiva latinoamericana reemplazada por el localismo; el desplazamiento de la preocupación por el sistema socioeconómico, sustituido por la centralidad del tema de la democracia; la negación del enfoque estructural y de proceso a través de una reducción situacional de la sociología. Esto se recoge en los siguientes párrafos sobre cada una de las consideraciones.

Sobre lo latinoamericano:

América Latina prácticamente ha desaparecido como objeto de estudio y ha sido reemplazada por una sociología de carácter nacional... Paradojalmente, este fenómeno de nacionalización de las ciencias sociales es un fenómeno latinoamericano cuyo carácter no se podrá entender porque, precisamente, en cada país se le considera un fenómeno nacional. En todo caso, hablar de América Latina hoy constituye casi una provocación para que de inmediato se descuelguen interminables listas de diferencias entre países. Hablar de América Latina es considerado lisa y llanamente una declaración de ignorancia... No se trata de llorar sobre la América Latina derramada, pero es evidente que su desaparición tiene hondas repercusiones en la temática sociológica. Al fin y al cabo, una buena parte de la sociología de la región giraba en torno de lo latinoamericano, del subdesarrollo, del tercermundismo, de la dependencia.

Sobre la forma democracia:

En general, la sociología nuestra siempre fue bastante política. Política en un doble sentido: porque se preocupaba de temas políticos y porque partía de una evaluación política. En este aspecto nos son escasas las amonestaciones que lanzaban los maestros del gran país del norte contra ese desmedido afán de politizarlo todo. Pues bien, 
esa es una característica que no se ha perdido y que, al contrario, se podría decir que es más fuerte. Sin embargo, ha cambiado el tema y ha cambiado el interés... Los estudios sociológicos de los últimos años han asumido una posición crítica frente al régimen autoritario que tiende a desplazar las antiguas preocupaciones por el sistema. En tal sentido, el tema de la democracia se ha tornado absolutamente preponderante, acompañado por piadosas consideraciones acerca de los sectores más desposeídos. A partir de aquí, la inspiración de los estudios sociológicos de lo político ha derivado fundamentalmente hacia dos vertientes. La una se manifiesta como condena de todo autoritarismo "venga de donde venga". La otra se vuelca a una valoración de lo popular que alcanza la grandeza del mito. Estas dos vertientes de inspiración de los estudios sociológicos tienden a confluir en una exaltada y a menudo confusa profesión de fe democrática en la cual se fundamentan sólidamente: la denuncia de la situación existente, la autocrítica del pasado y el silencio sobre el futuro. No se trata de cuestionar aquí la profesión de fe democrática que proclaman muchos estudios sociales del último tiempo. Al fin y al cabo, cada cual profesa la fe que le parece. Lo único que se desea señalar es que el abandono de las utopías sustantivas y su reemplazo por las soluciones formales es un fenómeno social del cual esos estudios constituyen un buen material de información, pero del cual no dan cuenta.

Sobre el enfoque situacional:

Otra característica que adquiere la sociología en el último tiempo es una fuerte atracción hacia la externalidad, hacia la apariencia más inmediata de los fenómenos sociales... Dos son las manifestaciones más repetidas de esta atracción por el inmediatismo apariencial. Por una parte, se produce un marcado interés por estudiar la relación entre lo privado y lo público, otorgando mayor relevancia al fenómeno privado directo. Por otra parte, se desarrolla considerablemente la preocupación por lo cotidiano, esto es, por los hechos rutinarios que no constituyen acontecimientos... Lo privado y lo cotidiano, no obstante las lucubraciones que se hagan al respecto, tienen, en relación al punto que nos interesa, la característica genérica de que aparecen como lo más inmediato. En este sentido, el Estado y las clases sociales constituyen conceptos de elevado nivel de abstracción que no son reductibles a la observación empírica directa. En cambio la vida cotidiana es lo que está ahí .Entendámonos. No se trata de que en un caso tengamos conceptos y en el otro no los tengamos. Eso, cuando menos, sería un absurdo. Pero lo que sí los diferencia es el nivel de la construcción conceptual. Particularmente porque, en el caso de la vida cotidiana, hay una definición situacional en cuanto expresión de un punto espacio temporal contiguo no extensible. La reducción situacional de la sociología niega la existencia de dos elementos que otrora se consideraban esenciales. Esto es, su carácter estructural, que estaba determinado por su concepción de totalidad; y su carácter de proceso, expresado en su connotación histórica. Este carácter situacional que tiende a adquirir la sociología conduce cada vez más a transformarla en una 
especie de periodismo. Los estudios escritos llegan, a veces, a ser de una contingencia tal que no resisten el paso de unos pocos meses. Gran parte de la producción sociológica, tiene la virtud de que, si no se la lee inmediatamente cuando aparece, ya no vale la pena leerla.

Las observaciones hechas en 1984, aunque preliminares y susceptibles de mucha crítica, están dando cuenta de una tendencia al cambio de temas y estilos que en la actualidad puede ser vista simplemente como los primeros indicios de un cambio de paradigmas que se desarrolla a nivel mundial y que corresponde a una nueva etapa en el desarrollo de la disciplina. Sin embargo, la expresión de que todo pensamiento es pensamiento de su época, ni siquiera en el más extremo idealismo significa que las ideas generen ideas, sino que estas se generan en situaciones históricas concretas. De manera que su especificidad puede ser relacionada con el Golpe militar de 1973, a partir de las condiciones que sustentan ese hecho político y de las condiciones que este a su vez produce. En tal sentido, y volviendo a nuestra especificidad, el ataque a la Universidad de Chile y a las ciencias sociales que ahí se desarrollaban, va a incidir fuertemente en los cambios de temas y estilos que estas experimentan, lo cual, incluso con la recuperación de la democracia, provocará inicialmente un aislamiento relativo con respecto a las ciencias sociales que se desarrollan en la región. Más aun, la hegemonía que determinadas perspectivas teóricas y posiciones ideológicas logran en el período autoritario se mantendrá y fortalecerá después de este sin encontrar resistencias de importancia. Un ejemplo importante al respecto es el predominio de formulaciones y estudios desde la óptica de la estratificación y la movilidad social, desplazando absolutamente las consideraciones sobre la conflictividad de clases.

Es posible que muchos estén de acuerdo en que se ha producido un cambio de temas y estilos en las ciencias sociales, pero la hegemonía también significa que muchos tienen una evaluación muy positiva de dichos cambios, los cuales pasan a ser considerados como una nueva etapa en el supuesto desarrollo evolutivo de las ciencias sociales, que dejaría en el pasado una etapa ideológica vinculada a unos obsoletos grandes relatos de sentido histórico.

\section{¿Y AHORA QUÉ?}

Han pasado ya cerca de 40 años desde el Golpe militar y más de 20 desde que concluyó el régimen que impuso violentamente. La Universidad de Chile, que fue pionera en el desarrollo de las ciencias sociales, tanto en investigación como en docencia, todavía no logra recuperarse de los efectos de la intervención militar, aunque realiza esfuerzos por su revitalización, como es el caso de la Iniciativa Bicentenario actualmente en curso.

Las ciencias sociales y particularmente la sociología que se desarrollaba en la Universidad de Chile, fueron definidas, por el Gobierno surgido del Golpe de Estado de 1973, en la categoría de enemigo y tratadas en consecuencia. De sus cultivadores, los que pudieron escapar a la persecución sufrieron un largo exilio del cual muchos no volvieron 
o se orientaron a otras actividades. En la Universidad Católica pudieron mantenerse ciertos estudios sociales, destacándose en 1979 la creación del Instituto de Ciencia Política. A su vez, la institucionalidad alternativa de las ONG creadas durante el Gobierno de Pinochet, fue hegemonizada por sociólogos formados en la Universidad Católica que, por tener mayor capital social, un perfil político más moderado o mayor proximidad a la Iglesia, lograron permanecer en el país o volver pronto a él. Esto se traduce también en el hecho de que los sociólogos más connotados a fines del siglo XX provengan mayoritariamente de esa Casa de Estudios.

En la Universidad de Chile la violencia del ataque y la represión produjo una ruptura, una discontinuidad, que no podía dejar de tener efectos en la recuperación de estas actividades. El ocaso del régimen militar permitió una lenta y difícil recuperación. Quienes habían logrado mantener la carrera tuvieron mayor libertad para resistir el rígido molde impuesto y unos pocos sociólogos de esta Universidad que habíamos podido permanecer en el país fuimos reincorporados. Se generaron así las condiciones para la revitalización de las ciencias sociales. En todo ello, y aunque parezca extraño, el factor fundamental fueron los estudiantes. Aun en las peores condiciones los estudiantes siempre impulsaron y apoyaron el desarrollo de una sociología crítica y comprometida como conciencia nacional. No solo sufrieron la represión directa, sino que buscaron, a través de prácticas autodidactas y de cursillos casi clandestinos con profesores expulsados, mantener una formación amplia y rigurosa y una apertura crítica al análisis social. Siempre y hasta el día de hoy, los jóvenes que eligen estudiar ciencias sociales en la Universidad de Chile son los mejores de su generación, no solo medidos por la vara de las pruebas de admisión, sino porque su orientación es fundamentalmente al conocimiento social y solo secundariamente a la obtención de un título profesional.

Naturalmente, es sabido que el régimen de Pinochet no solo atacó a las ciencias sociales de la Universidad de Chile, sino que a esta Universidad en su conjunto. Si no logró eliminarla por completo, al menos logró reducirla radicalmente al arrebatarle sus sedes regionales y el Pedagógico, reduciendo sus matrículas a la mitad y obligándola al autofinanciamiento privatizador. Distinto fue el trato con la Universidad Católica, a la cual solo se intentó depurarla de los elementos considerados negativos y desde donde salieron economistas y cuadros políticos importantes para la implantación del nuevo modelo. Estas diferencias de trato están en la base de las distintas capacidades de competir de estas universidades, que van en desmedro de la Universidad de Chile y que se complicarán mucho más con el fuerte impulso dado a la creación y masificación de nuevas universidades privadas.

Pero no se pretende aquí simplemente señalar las transformaciones ocurridas en el sistema universitario y que son suficientemente conocidas. Lo que se intenta es mostrar la manera en que históricamente y de una manera concreta se producen esas transformaciones, para así tratar de interpretar lo ocurrido en las ciencias sociales y particularmente en la sociología. En efecto, sería absurdo pretender explicar los cambios ocurridos en los estudios de sociología en Chile simplemente como una consecuencia del Golpe militar de 1973. Pero también resulta absurdo explicar dichos cambios como consecuencia de una transformación a nivel mundial de las ideas sobre la sociología. El 
Golpe militar en Chile es una singularidad, pero es una singularidad que se entiende en su vinculación con grandes transformaciones a nivel global de las cuales recién estamos tomando conciencia. A su vez, esas grandes transformaciones solo son posibles en tanto se realizan en singularidades concretas que establecen las condiciones de su desarrollo.

Si contemplamos el actual panorama de la sociología en Chile veremos que en líneas generales no difiere de lo que ocurre en otras latitudes, aunque por cierto hay especificidades. En Chile aparece más efectivamente marcada una creciente profesionalización de los estudios de sociología, que van acompañados por una tecnificación del quehacer sociológico y una preocupación por temas y problemas específicos. Esta profesionalización de la carrera queda simbólicamente establecida cuando el Gobierno de Pinochet quita el carácter universitario que tenía la sociología y la declara una carrera que también pueden impartir los institutos profesionales. Más allá de pronunciamientos formales, es posible apreciar que se produce una pérdida de visión generalizadora hacia lo que alguna vez fueron grandes temas como América Latina, estructura social, ideologías, cambio social, procesos. A nivel de los países centrales, persiste todavía la elaboración de grandes relatos de interpretación del cambio social, aunque paradojalmente estos grandes relatos se planteen desde el supuesto del fin de los grandes relatos.

Ante este panorama, resulta interesante recordar que hace algunos años un conocido sociólogo, formado en la Pontificia Universidad Católica y montado en ese entonces en un cargo ministerial, proclamó enfáticamente el fin de la sociología. El argumento era simple. Hay dos estilos de desarrollo de la sociología, uno proveniente de la epopeya, que genera una perspectiva de sistemas y grandes relatos; el otro minimalista, asociado a la novela con sus pequeños conceptos de interpretación de la vida cotidiana. Desde su perspectiva, ninguna de estas dos sociologías tiene actualmente sentido, ya que han desaparecido los grandes relatos, y la novela y los medios de comunicación dan mejor cuenta de la vida cotidiana.

Frente a la fuerza de esas narrativas, la sociología -en sus vertientes macro y micropareciera ir quedando fuera de la escena intelectual y del marco comunicativo. Su familiaridad con lo actual es escasa; sus reconstrucciones epopéyicas han sido consumidas; su perplejidad ante el mundo es menos rica, variada y auténtica que aquella de la novela o de las artes audiovisuales. Quizás sea cierto entonces que el universo ideológico-lingüístico de nuestra profesión esté en vías de desaparición, ahora que los "grandes relatos" parecen haberse desacreditado y las micro-representaciones de la vida cotidiana se hallan mejor servidas por los medios de comunicación ${ }^{13}$.

Dado que ya se ha decretado el fin de las clases sociales, el fin del Estado Nación, el fin de las ideologías y hasta el fin de la historia, que alguien declare el fin de la sociología no es como para conmoverse. Sin embargo, las declaraciones enfáticas tienen el mérito de hacer más fáciles los análisis. La simplificación de la sociología en términos de grandes

13. Brunner, J.J., "Sobre el crepúsculo de la sociología y el comienzo de otras narrativas", en Celebración de los cuarenta años de FLACSO en América Latina, FLACSO-Chile, 1997. 
relatos y descripción de la vida cotidiana, ignora algo que es esencial en esta disciplina, que es la intención de hacer consciente lo social y tratar de comprenderlo.

Algunos plantean el carácter crítico del pensamiento sobre lo social, entendiéndolo como un develamiento de algo que debe ser cambiado, como la capacidad de hacer consciente a la sociedad de una situación negativa que mediante esa conciencia puede llegar a ser cambiada. Pero no es claro que el conocimiento de lo social tenga que orientarse a la crítica de un estado de cosas e impulsar alternativas de cambio, también puede orientarse a su conservación. Lo que sí es cierto es que el desconocimiento de lo social permite que este se presente como lo natural evitando toda evaluación del mismo.

Todo pensamiento es pensamiento de su época. Durante los años 60 se produjo una espectacular expansión de la sociología. En la Universidad de Chile se incorporó como curso formativo en casi todas las carreras y en muchas de ellas se agregó cursos de sociología especializada en la disciplina (hubo sociología médica, sociología jurídica, sociología urbana, sociología económica). Eran momentos de agitación social y política, de crisis en el verdadero sentido de la palabra, que indica un momento de encrucijada donde hay que decidir un camino. Los problemas exigían una comprensión y análisis de lo social, mientras las ideologías orientaban las búsquedas.

La consideración del fin de la sociología, casi tiene el carácter de una propuesta. Ya no es necesaria, porque ha finalizado la historia y no tienen sentido los grandes relatos ni utopías y porque la industria cultural y los medios de comunicación nos proporcionan la adecuada interpretación de lo cotidiano. Todo pensamiento es pensamiento de su época. A fines de siglo se consolidaba un modelo económico y un modelo político de carácter global, parecía ya no haber problemas que reclamaran un conocimiento de lo social. Como señalaba ese mismo autor, las grandes categorías sistémicas ya no resisten los embates del Banco Mundial, donde se ordena todo. En esta perspectiva, la sociología solo tiene sentido como una profesión técnica que proporcione las herramientas y conocimientos para una administración más eficiente de los recursos públicos o de las empresas.

Pero el tiempo tiene esa porfía tan suya de seguir corriendo. Y si el amor es eterno mientras dura, también las épocas son estables mientras no se alteran.

\section{REFERENCIAS BIBLIOGRÁFICAS}

Alvarado, M., Santander, P., "Matar al padre': Análisis discursivo de dos textos de la sociología chilena en período de dictadura", en Literatura y Lingüistica, 14, UCSH, 2003.

Archivo Chile, Sumariados, "La dictadura y la educación", véase en www.archivochile.cl

Arzobispado de Santiago, Fundación de documentación y archivo de la Vicaría de la Solidaridad, Capítulo quinto: "La situación laboral", No hay más referencias.

Baño, R,. "Nuevos estilos y nuevos temas en el análisis de ciencias sociales en la última década", Documento de Discusión, No 60, Programa FLACSO, Santiago, 1984.

Brunner, J.J., Barrios, A., El caso de la sociología en Chile. Formación de una disciplina, Santiago: FLACSO, 1988. 
Brunner, J.J., "Sobre el crepúsculo de la sociología y el comienzo de otras narrativas", en Celebración de los cuarenta años de FLACSO en América Latina, FLACSO-Chile, 1997.

Comisión Nacional de Verdad y Reconciliación, Colección Archivo Nacional de Chile, Santiago, 1991.

Fernández, M. de los A., "Ciencia Política en Chile: Un espejo intelectual", en Revista de Ciencia Politica, 25 (1), Pontificia Universidad Católica de Chile, 2005.

Garretón, M.A., "Las Ciencias Sociales en Chile. Institucionalización, ruptura y renacimiento, véase www.manuelantoniogarreton.cl/ultimosl.html

Vasconi, T.A., "Las ciencias sociales en América del Sur y Chile, 1960-1990", Working Paper Centro de Investigaciones Sociales, Universidad ARCIS, 1996. 